\title{
Glucocorticoid treatment impairs microvascular function in healthy men in association with its adverse effects on glucose metabolism and blood pressure: a randomised controlled trial
}

\author{
Daniël H. van Raalte • Michaela Diamant • D. Margriet Ouwens • \\ Richard G. Ijzerman • Margot M. L. Linssen • Bruno Guigas • \\ Etto C. Eringa • Erik H. Serné
}

Received: 30 April 2013 / Accepted: 15 July 2013 /Published online: 11 August 2013

(C) Springer-Verlag Berlin Heidelberg 2013

\begin{abstract}
Aims/hypothesis Glucocorticoids (GCs) are widely used antiinflammatory agents that frequently induce side effects, including insulin resistance, diabetes and hypertension. Here, we investigated the contribution of microvascular dysfunction to the development of these adverse effects in healthy men.

Methods In a randomised, placebo-controlled, dose-response intervention study, 32 healthy normoglycaemic men (age: $21 \pm 2$ years; BMI: $21.9 \pm 1.7 \mathrm{~kg} / \mathrm{m}^{2}$ ) were allocated to receive prednisolone $30 \mathrm{mg}$ once daily $(n=12)$, prednisolone $7.5 \mathrm{mg}$ once daily $(n=12)$ or placebo $(n=8)$ for 2 weeks using block randomisation. A central office performed the treatment allocation, and medication was dispersed by the hospital pharmacy that was also blinded. Treatment allocation was kept in concealed envelopes. Participants, study personnel conducting
\end{abstract}

Electronic supplementary material The online version of this article (doi:10.1007/s00125-013-3016-8) contains peer-reviewed but unedited supplementary material, which is available to authorised users.

D. H. van Raalte $(\bowtie) \cdot$ M. Diamant $•$ R. G. Ijzerman • E. H. Serné Diabetes Center, VU University Medical Center, de Boelelaan 1117, 1081 HV Amsterdam, the Netherlands

e-mail: d.vanraalte@vumc.nl

D. M. Ouwens

Institute of Clinical Biochemistry and Pathobiochemistry, German Diabetes Center, Düsseldorf, Germany

M. M. L. Linssen • B. Guigas

Department of Molecular Cell Biology, Leiden University Medical

Center, Leiden, the Netherlands

E. C. Eringa

Laboratory for Physiology, Institute for Cardiovascular Research, VU University Medical Center, Amsterdam, the Netherlands the measures and assessing the outcome were blinded to group assignment. The study was conducted at a university hospital. Primary endpoint was prednisolone-induced changes in microvascular function, which was assessed by capillary microscopy. Insulin sensitivity was determined by hyperinsulinaemiceuglycaemic clamp and postprandial glycaemic excursions by standardised meal tests.

Results Compared with placebo, prednisolone $7.5 \mathrm{mg}$ and $30 \mathrm{mg}$ decreased insulin-stimulated capillary recruitment by $9 \pm 4 \%$ and $17 \pm 3 \%$, respectively $(p<0.01)$. In addition, prednisolone $7.5 \mathrm{mg}$ and $30 \mathrm{mg}$ reduced insulin sensitivity ( $M$ value) by $-11.4 \pm 4.5 \mu \mathrm{mol} \mathrm{kg}{ }^{-1} \mathrm{~min}^{-1}$ and $-25.1 \pm 4.1 \mu \mathrm{mol} \mathrm{kg}^{-1} \min ^{-1}$ $(p<0.001)$ and increased postprandial glucose levels by $11 \pm 5 \%$ and $27 \pm 9 \%(p<0.001)$, respectively. Only high-dose prednisolone increased systolic blood pressure $(6 \pm 1.2 \mathrm{mmHg}$, $p=0.006$ ). Prednisolone-induced changes in insulin-stimulated capillary recruitment were associated with insulin sensitivity $(r=+0.76 ; p<0.001)$, postprandial glucose concentrations $(r=-0.52 ; p<0.03)$ and systolic blood pressure $(r=-0.62$; $p<0.001)$. Prednisolone increased resistin concentrations, which were negatively related to insulin-stimulated capillary recruitment $(r=-0.40 ; p=0.03)$. No effects were noted on adiponectin and leptin concentrations. Prednisolone treatment was well tolerated; none of the participants left the study.

Conclusions/interpretation Prednisolone-induced impairment of insulin-stimulated capillary recruitment was paralleled by insulin resistance, increased postprandial glucose levels, hypertension and increased circulating resistin concentrations in healthy men. We propose that GC-induced impairments of microvascular function may contribute to the adverse effects of GC treatment on glucose metabolism and blood pressure. Trial registration isrctn.org ISRTCN 78149983

Funding The study was funded by the Dutch Top Institute Pharma T1-106. 
Keywords Adipokines $\cdot$ Glucocorticoids $\cdot$ Hypertension . Insulin resistance $\cdot$ Microvascular function $\cdot$ Steroid diabetes

$\begin{array}{ll}\text { Abbreviations } \\ \text { AUC }_{\mathrm{G}} & \text { AUC for postprandial glucose curve } \\ \text { eNOS } & \text { Endothelial nitric oxide synthase } \\ \text { FPG } & \text { Fasting plasma glucose } \\ \text { GC } & \text { Glucocorticoid } \\ \text { GIP } & \text { Glucose-dependent insulinotropic peptide } \\ \text { GLP } & \text { Glucagon-like peptide } \\ \text { NO } & \text { Nitric oxide } \\ \text { OGIS } & \text { Oral glucose insulin sensitivity }\end{array}$

\section{Introduction}

Glucocorticoids (GCs) represent the most important and most commonly prescribed class of anti-inflammatory drugs, but their use is hampered by an increased risk of serious side effects, particularly hypertension, insulin resistance, impaired glucose tolerance and overt diabetes in susceptible individuals [1-5]. In addition, GCs promote visceral adipose tissue deposition and change adipose tissue biology resulting in enhanced lipolysis [6] and altered secretion of a number of adipose tissue-derived hormones and cytokines in vitro [7]. Although the exact mechanisms underlying these adverse effects remain to be identified, GC-induced insulin resistance may underlie part of these side effects [6]. Insulin resistance is characterised by the diminished ability of insulin to initiate intracellular signalling, primarily in the liver, adipose tissue and skeletal muscle [8]. Impaired insulin signalling in these tissues results in reduced glucose uptake, insufficient suppression of hepatic glucose output, compensatory hyperinsulinaemia and dyslipidaemia, which collectively constitute the so-called metabolic syndrome [8].

Insulin action is not restricted to metabolically active tissues. Recently, it has become clear that vascular tissue (particularly endothelial cells) represents an important physiological target for insulin and is a significant regulator of overall insulin-stimulated glucose uptake [9-11]. Insulin promotes its own access to muscle interstitial space by increasing blood flow and by recruiting capillaries to expand the endothelial transporting surface available for nutrient exchange [10]. In addition, the local actions of insulin on the diameter of resistance arteries may be of relevance to the pathogenesis of hypertension, an important feature of the metabolic syndrome $[9,11-13]$. Insulin exerts a vasodilatory action by promoting the release of nitric oxide (NO) from endothelial cells. This involves the phosphorylation of endothelial NO synthase (eNOS), which in turn is mediated by the phosphatidylinositol 3-kinase/Akt pathway [14]. In insulin-resistant states, however, insulin-stimulated vasodilatation and capillary recruitment become compromised, and this may contribute to the development of impaired whole-body insulin-stimulated glucose uptake and hypertension [15]. The mechanism by which capillary recruitment is impaired in insulin-resistant states is presently unknown, although changes in secretion of adipose-tissue-derived factors, possibly from perivascular fat depots, may be involved [16, 17].

It is presently unclear whether vascular insulin resistance could be involved in the development of the unfavourable effects of GCs on glucose metabolism and blood pressure. Thus, in the present study, we investigated whether the metabolic effects of the GC prednisolone, given at both a clinically relevant low and high dosage for 2 weeks, would concomitantly induce vascular insulin resistance, defined as impaired insulin-stimulated capillary recruitment, in healthy men. Subsequently, we assessed associations between prednisolone-induced vascular insulin resistance and changes in blood pressure, whole-body insulin-mediated glucose uptake and postprandial glucose levels. Finally, we examined whether alterations in adipokine levels are related to changes in microvascular function.

\section{Methods}

Participants Thirty-two healthy white men were recruited by local advertisement. Inclusion criteria were age 18-35 years, BMI $20.0-25.0 \mathrm{~kg} / \mathrm{m}^{2}$, good physical health (determined by medical history, physical examination and screening blood tests) and normoglycaemia as defined by fasting plasma glucose $(\mathrm{FPG})<5.6 \mathrm{mmol} / \mathrm{l}$ and $2 \mathrm{~h}$ glucose $<7.8 \mathrm{mmol} / \mathrm{l}$ following a $75 \mathrm{~g}$ OGTT, performed at screening visit. Exclusion criteria were any current illness, use of any medication, firstdegree relative with type 2 diabetes, smoking, shift work, a history of GC use, excessive sports activity (i.e. more often than twice a week) and recent changes in weight or physical activity. The study was approved by an independent ethics committee and was conducted in accordance with the Declaration of Helsinki. All participants provided written informed consent before participation.

Study design The study was a randomised, placebo-controlled, double-blind, dose-response intervention study. Following assessment of eligibility and baseline measurements, participants were randomised to receive either prednisolone $30 \mathrm{mg}$ once daily $(n=12)$, prednisolone $7.5 \mathrm{mg}$ once daily $(n=12)$ or placebo treatment $(n=8)$ for a period of 14 days using block randomisation, as carried out by the Department of Experimental Pharmacology of the VU University Medical Center. Before treatment and on day 13 of treatment, microvascular function was measured by capillary microscopy in the fasted state and during insulin infusion. Insulin sensitivity was 
determined by hyperinsulinaemic-euglycaemic clamp. Before treatment and on day 14 of treatment, a standardised consecutive meal challenge test was performed to assess glucose tolerance. All measurements were conducted in a temperature-controlled room $\left(23.4 \pm 0.4^{\circ} \mathrm{C}\right)$ starting at 07:30 hours, following a $12 \mathrm{~h}$ overnight fast with the participants in the supine position. Participants refrained from drinking alcohol for a period of $24 \mathrm{~h}$ before the study day and did not perform strenuous exercise for a period of $48 \mathrm{~h}$ before each study day. Baseline measurements were obtained following $30 \mathrm{~min}$ of rest and acclimatisation. During all visits, including a follow-up visit on day 7 of treatment, safety and tolerability were assessed (see electronic supplementary material [ESM] Fig. 1). A patient flow diagram is shown in Fig. 1.

Capillary microscopy Nail-fold capillary studies were performed in the fasted state $(\mathrm{T}=-120 \mathrm{~min})$ and during hyperinsulinaemia $(\mathrm{T}=120 \mathrm{~min})$ as described previously [18] by a single experienced investigator (ESM Fig. 2). Baseline capillary density was defined as the number of continuously erythrocyte-perfused capillaries per $\mathrm{mm}^{2}$ of nail-fold skin. Post-occlusive reactive hyperaemia after 4 min of arterial occlusion was used to assess functional capillary recruitment. Capillary recruitment was calculated by dividing the increase in perfused capillary density during post-occlusive reactive hyperaemia by the baseline perfused capillary density. In addition, venous congestion to expose a maximal number of capillaries was carried out. All procedures were performed twice, and the mean of both measurements was used for analyses. The day-to-day coefficient of variation of functional capillary recruitment was $15.9 \pm 8.0 \%$ as determined in 10 healthy individuals on two separate days. Skin temperature was monitored during the tests. One participant in the prednisolone $7.5 \mathrm{mg}$ arm could not be analysed due to insufficient quality of microscopic images during treatment with study medication and was left out of the analyses.

Blood pressure measurement Systolic and diastolic blood pressure were manually measured (Welch Allyn, Delft, the Netherlands) by a single experienced investigator following the 30 min acclimatisation period. The average of three consecutive blood pressure measurements with a 5 min interval was used for further analyses.

Hyperinsulinaemic-euglycaemic clamp Whole-body insulin sensitivity was assessed by the hyperinsulinaemic-euglycaemic clamp method as described previously [19] with insulin infusion initiated at $\mathrm{T}=0$ (ESM Fig. 2). Briefly, a primed, continuous $\left(40 \mathrm{mU} \mathrm{m}^{-2} \mathrm{~min}^{-1}\right.$ ) insulin infusion (Actrapid $100 \mathrm{IU} / \mathrm{ml}$; Novo Nordisk, Bagsvaerd, Denmark) was given for 120 min; plasma glucose concentration was kept at $5 \mathrm{mmol} / \mathrm{l}$ by adjusting the rate of a $20 \%$ glucose infusion based on plasma glucose measurements performed at $5 \mathrm{~min}$ intervals (Fig. 1). Whole-body insulin sensitivity was quantified by the $M$ value $\left(\mu \mathrm{mol} \mathrm{kg}{ }^{-1} \mathrm{~min}^{-1}\right)$, calculated between 90 and 120 min during steady-state insulin

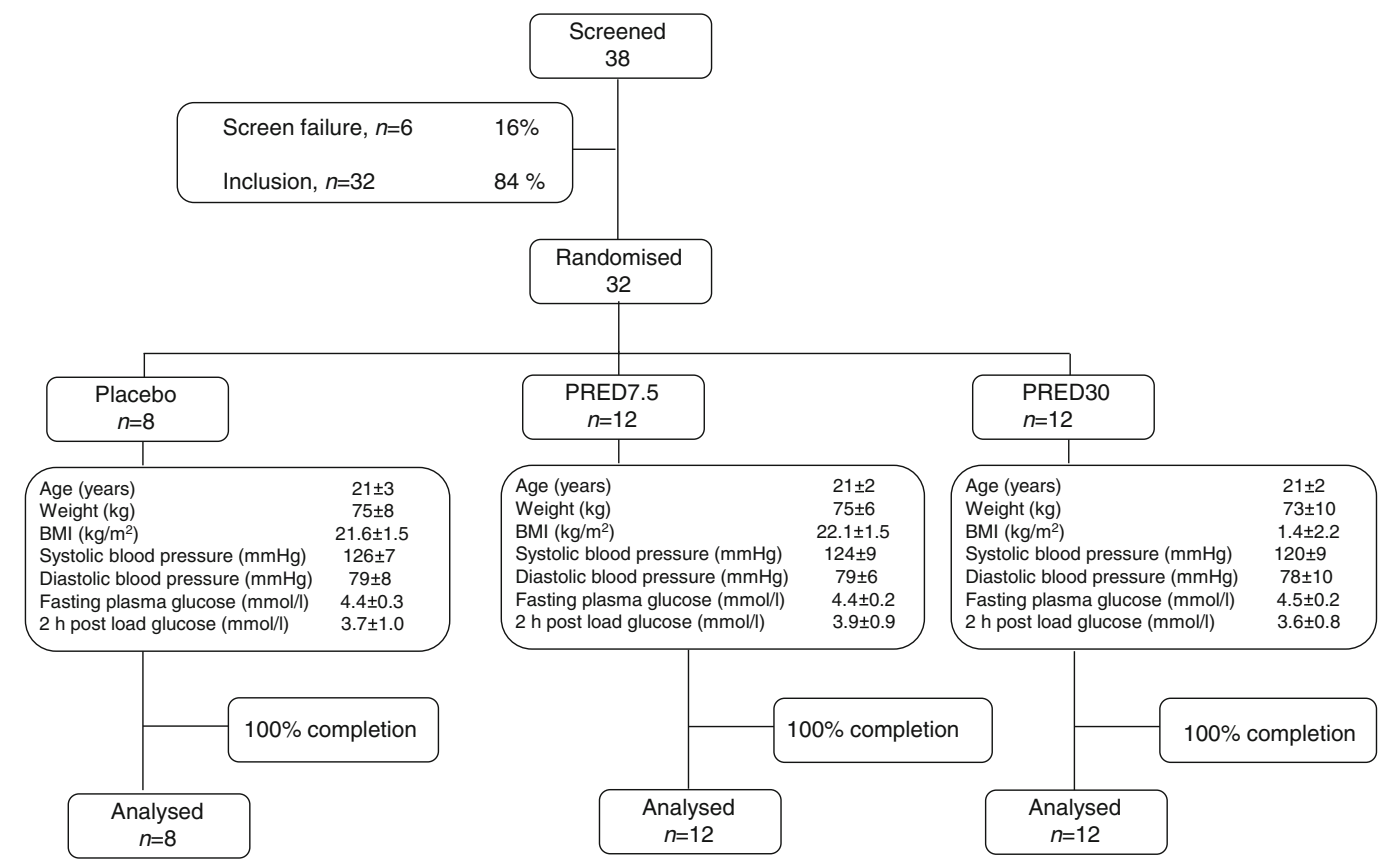

Fig. 1 Patient flow diagram. One-hundred per cent of the randomised participants completed the study. Reasons for screen failures $(n=6)$ were: one individual was a smoker, one individual had a chronic inflammatory condition, two individuals engaged in physical activity $>3$ times per

week, one individual had a first-degree relative with type 2 diabetes and one individual had abnormal laboratory values. PRED7.5, participants received $7.5 \mathrm{mg}$ prednisolone daily for 14 days; PRED30, participants received $30 \mathrm{mg}$ prednisolone daily for 14 days 
concentrations, as described previously [19]. Insulin levels were determined at $30 \mathrm{~min}$ intervals before and during the clamp as shown in ESM Fig. 2.

Standardised meal test Postprandial glucose metabolism was assessed following two consecutive identical meals (breakfast at 09:00 hours and lunch at 13:00 hours). The meals contained $3,787 \mathrm{~kJ}$ (50 g fat, $75 \mathrm{~g}$ carbohydrates, $35 \mathrm{~g}$ protein) each. Samples for determination of glucose were obtained at $30 \mathrm{~min}$ intervals, with the meal beginning immediately after the time 0 sample and consumed within $15 \mathrm{~min}$. Area under the postprandial glucose curve $\left(\mathrm{AUC}_{\mathrm{G}}\right)$ was calculated by the trapezoid method. Insulin sensitivity in the postprandial state was calculated as the oral glucose sensitivity index (OGIS) [20].

Study medication Prednisolone tablets were purchased from Pfizer (Sollentuna, Sweden) and placebo tablets were obtained from Xendo Drug Development (Groningen, the Netherlands). Tablets were encapsulated to allow the treatment to be blinded [21]. Study medication was taken at 08:00 hours during the 2-week treatment except for day 13 and day 14, when it was ingested at 06:00 hours. Patients kept a diary in which the exact time of medication intake during the study was recorded.

Biochemical analyses Blood glucose concentrations were measured using a YSI 2300 STAT Plus analyser (YSI, Yellow Springs, OH, USA). Insulin levels were determined using an immunometric assay (Advia, Centaur; Siemens Medical Solutions Diagnostics, Tarrytown, NY, USA). Glucagon concentrations were determined by radioimmunoassay (Linco Research, St Louis, MO, USA). Total glucagon-like peptide (GLP)-1 levels and glucose-dependent insulinotropic peptide (GIP) levels were measured using radioimmunoassays as described previously $[22,23]$. A single-plex human magnetic adiponectin and a duo-plex human leptin and resistin assay (all from Biorad, Hercules, CA, USA) were used to determine serum levels of adiponectin, leptin and resistin using a Bioplex 200 suspension array system and Bioplex Pro II wash station (Biorad), respectively. Adipokine concentrations were calculated from the appropriate optimised standard curves using Bio-Plex Manager software version 6.0 (Biorad). Intra- and interassay coefficients of variation for the Bioplex assays were both $<4 \%$.

Statistical analyses Data are presented as means $\pm \mathrm{SD}$, or as median (interquartile range) in case of skewed distribution. Absolute changes from baseline were calculated (on treatment value minus pre-treatment value) for all variables. Nonparametric analysis was chosen due to uneven sample size in the different groups and the relatively small numbers in each group. Baseline values were compared between groups by the
Kruskal-Wallis test. For treatment-induced effects, KruskalWallis with trend analysis was performed ("the JonckheereTerpstra test'). Only in the case of a significant finding were prednisolone $7.5 \mathrm{mg}$ and prednisolone $30 \mathrm{mg}$ compared against placebo by post hoc testing, using the Mann-Whitney $U$ test. To correct for multiple testing, Bonferroni correction was applied. Correlations between variables were assessed with Spearman correlations. All statistical analyses were run using SPSS version 18.0 for Mac OS X (Chicago, IL, USA). $p<0.05$ was considered statistically significant.

\section{Results}

Baseline characteristics and anthropometrics Before treatment, all treatment groups had comparable age, anthropometrics, blood pressure and glucose tolerance (Table 1).

Prednisolone dose-dependently impairs glucose metabolism and insulin sensitivity Prednisolone $30 \mathrm{mg}$, but not $7.5 \mathrm{mg}$, increased FPG levels relative to placebo $(p=0.04)$, despite fasting hyperinsulinaemia $(p=0.008)$ (Table 2$)$. As compared with placebo, glucose levels during the standardised meal test were dose-dependently increased by prednisolone treatment: glucose levels (measured as AUC) increased by $11 \pm 5 \%$ and $27 \pm 9 \%$, for prednisolone $7.5 \mathrm{mg}$ and prednisolone $30 \mathrm{mg}$, respectively (Table 2). Clamp-measured whole-body insulin sensitivity was decreased by prednisolone treatment as compared with placebo in a dose-dependent manner: mean difference in $M$ value was $-11.4 \pm 4.5 \mu \mathrm{mol} \mathrm{kg}^{-1} \mathrm{~min}^{-1}$ for prednisolone $7.5 \mathrm{mg}$ and $-25.1 \pm 4.1 \mu \mathrm{mol} \mathrm{kg}^{-1} \mathrm{~min}^{-1}$ for prednisolone

Table 1 Baseline characteristics

\begin{tabular}{|c|c|c|c|c|}
\hline \multirow[t]{2}{*}{ Characteristic } & \multirow[t]{2}{*}{ Placebo } & \multicolumn{2}{|c|}{ Prednisolone } & \multirow[t]{2}{*}{$p$ value } \\
\hline & & $7.5 \mathrm{mg}$ & $30 \mathrm{mg}$ & \\
\hline$n$ & 8 & 12 & 12 & \\
\hline Age (years) & $21 \pm 3$ & $21 \pm 2$ & $21 \pm 2$ & 0.670 \\
\hline Weight (kg) & $75 \pm 8$ & $75 \pm 6$ & $73 \pm 10$ & 0.886 \\
\hline Height (cm) & $186 \pm 7$ & $184 \pm 6$ & $185 \pm 5$ & 0.718 \\
\hline BMI $\left(\mathrm{kg} / \mathrm{m}^{2}\right)$ & $21.6 \pm 1.5$ & $22.1 \pm 1.5$ & $21.4 \pm 2.2$ & 0.656 \\
\hline $\begin{array}{l}\text { Systolic blood pressure } \\
(\mathrm{mmHg})\end{array}$ & $126 \pm 7$ & $124 \pm 9$ & $120 \pm 9$ & 0.284 \\
\hline $\begin{array}{l}\text { Diastolic blood pressure } \\
(\mathrm{mmHg})\end{array}$ & $79 \pm 8$ & $79 \pm 6$ & $78 \pm 10$ & 0.872 \\
\hline FPG $(\mathrm{mmol} / \mathrm{l})$ & $4.4 \pm 0.3$ & $4.4 \pm 0.2$ & $4.5 \pm 0.2$ & 0.852 \\
\hline 2 h OGTT $(\mathrm{mmol} / \mathrm{l})$ & $3.7 \pm 1.0$ & $3.9 \pm 0.9$ & $3.6 \pm 0.8$ & 0.782 \\
\hline
\end{tabular}

Data are means $\pm \mathrm{SD}$

No statistically significant differences were observed between the groups at baseline. $2 \mathrm{~h}$ OGTT denotes plasma glucose concentrations $2 \mathrm{~h}$ after ingestion of $75 \mathrm{~g}$ glucose 
$30 \mathrm{mg}$. Similarly, postprandial insulin sensitivity, calculated by OGIS, was dose-dependently reduced by prednisolone treatment as compared with placebo (Table 2). Treatment with study medication did not alter body weight (Table 2). While postprandial glucagon levels were increased by prednisolone treatment, plasma levels of incretin hormones (GLP-1 and GIP) were unaltered (Table 2).

Prednisolone increases systolic blood pressure Systolic blood pressure, but not diastolic blood pressure, was raised by prednisolone $30 \mathrm{mg}(6 \pm 1.2 \mathrm{mmHg}, p=0.006$ vs placebo), whereas prednisolone $7.5 \mathrm{mg}$ did not affect blood pressure (Table 2).

Prednisolone impairs capillary recruitment Prednisolone treatment did not affect baseline capillary density, capillary recruitment or maximal number of capillaries following venous congestion in the fasted state (Fig. 2a, Table 3). Insulin infusion increased capillary recruitment by $13 \pm 4 \%(p<0.001)$ but did not affect baseline capillary density (Table 3 ). During insulin infusion, prednisolone treatment did not affect baseline capillary density, although it dose-dependently reduced functional capillary recruitment by $9 \pm 4 \%$ (prednisolone $7.5 \mathrm{mg}$ ) and $17 \pm 3 \%$ (prednisolone $30 \mathrm{mg}$ ) as compared with placebo (Fig. 2b, Table 3).


Fig. 2 Effect of prednisolone on capillary recruitment (CR). CR in the fasted state was not impaired by either of the prednisolone doses (a), although prednisolone treatment dose-dependently impaired insulin-stimulated microvascular function (b). Data represent absolute change from baseline (mean \pm SEM). White bars, placebo; grey bars, prednisolone $7.5 \mathrm{mg}$ daily; black bars, prednisolone $30 \mathrm{mg}$ daily. $* * * p<0.001$ across groups; $* * p<0.01$ prednisolone $30 \mathrm{mg}$ daily vs placebo

Inter-relationship of insulin-stimulated capillary recruitment, metabolic variables and blood pressure Prednisolone-induced reductions in insulin-stimulated capillary recruitment were negatively correlated with changes in fasting $(p<0.0001)$ (Fig. 3a) and postprandial glucose levels ( $p=0.003$ ) (Fig. 3b), while positive associations were found with impaired clampmeasured $(p<0.0001)$ (Fig. 3c) and postprandial insulin sensitivity ( $p=0.004$ ) (Fig. 3d). In multiple regression analyses, adjusting for age and BMI, changes in insulin-stimulated capillary recruitment statistically explained approximately

Table 2 Metabolic variables and blood pressure before and during treatment

\begin{tabular}{|c|c|c|c|c|c|c|c|c|c|}
\hline \multirow[t]{2}{*}{ Variable } & \multicolumn{2}{|l|}{ Placebo } & \multicolumn{2}{|c|}{ Prednisolone $7.5 \mathrm{mg}$ daily } & \multicolumn{2}{|c|}{ Prednisolone $30 \mathrm{mg}$ daily } & \multirow[t]{2}{*}{$p 1$} & \multirow[t]{2}{*}{$p 2$} & \multirow[t]{2}{*}{$p 3$} \\
\hline & Baseline & On treatment & Baseline & On treatment & Baseline & On treatment & & & \\
\hline Weight (kg) & $75 \pm 8$ & $76 \pm 7$ & $75 \pm 6$ & $75 \pm 6$ & $73 \pm 10$ & $74 \pm 10$ & 0.539 & NA & NA \\
\hline SBP $(\mathrm{mmHg})$ & $126 \pm 7$ & $123 \pm 9$ & $124 \pm 9$ & $124 \pm 11$ & $120 \pm 9$ & $124 \pm 8$ & 0.002 & 0.710 & 0.006 \\
\hline DBP (mmHg) & $79 \pm 8$ & $76 \pm 7$ & $79 \pm 6$ & $76 \pm 6$ & $78 \pm 10$ & $80 \pm 9$ & 0.042 & 1.0 & 0.164 \\
\hline FPG (mmol/l) & $4.4 \pm 0.3$ & $4.5 \pm 0.3$ & $4.4 \pm 0.2$ & $4.7 \pm 0.3$ & $4.5 \pm 0.2$ & $4.9 \pm 0.4$ & 0.04 & 0.418 & 0.09 \\
\hline FPI (pmol/l) & $31(28-40)$ & $33(28-39)$ & $36(29-47)$ & $36(27-65)$ & $32(26-44)$ & $56(41-72)$ & 0.001 & 1.0 & 0.008 \\
\hline $\mathrm{AUC}_{\mathrm{G}}(\mathrm{mmol} / 1 \times \mathrm{h})$ & $2,197 \pm 193$ & $2,182 \pm 92$ & $2,250 \pm 121$ & $2,492 \pm 127$ & $2,189 \pm 228$ & $2,734 \pm 260$ & $<0.0001$ & 0.008 & 0.002 \\
\hline $\operatorname{AUC}_{\mathrm{GLG}}(\mathrm{nmol} / 1 \times \mathrm{h})$ & $6.9 \pm 1.6$ & $6.5 \pm 1.5$ & $7.2 \pm 1.7$ & $7.4 \pm 1.6$ & $6.6 \pm 1.2$ & $8.4 \pm 2.0$ & 0.002 & 0.49 & 0.002 \\
\hline $\mathrm{AUC}_{\mathrm{GLP}-1}(\mathrm{nmol} / \mathrm{l} \times \mathrm{h})$ & $17.0 \pm 3.1$ & $16.6 \pm 3.8$ & $16.1 \pm 2.5$ & $16.5 \pm 3.9$ & $15.1 \pm 3.4$ & $15.8 \pm 2.4$ & 0.575 & NA & NA \\
\hline $\operatorname{AUC}_{\mathrm{GIP}}(\mathrm{nmol} / \mathrm{l} \times \mathrm{h})$ & $36.6 \pm 12.6$ & $40.8 \pm 9.3$ & $40.5 \pm 9.6$ & $46.9 \pm 16.0$ & $45.3 \pm 12.3$ & $53.3 \pm 17.2$ & 0.869 & NA & NA \\
\hline OGIS $\left.\left(\mathrm{ml} \mathrm{min} \min ^{-1} \mathrm{~m}^{-2}\right)\right)$ & $488 \pm 50$ & $489 \pm 33$ & $477 \pm 41$ & $420 \pm 42$ & $484 \pm 52$ & $375 \pm 75$ & $<0.0001$ & 0.01 & 0.004 \\
\hline$M$ value $\left(\mu \mathrm{mol} \mathrm{kg}{ }^{-1} \min ^{-1}\right)$ & $39(26-62)$ & $50(36-71)$ & $51(41-59)$ & $51(25-62)$ & $42(32-52)$ & $26(16-34)$ & $<0.0001$ & 0.062 & $<0.001$ \\
\hline Clamp glucose (mmol/l) & $5.0 \pm 0.3$ & $5.0 \pm 0.2$ & $5.0 \pm 0.1$ & $5.0 \pm 0.3$ & $5.0 \pm 0.1$ & $5.1 \pm 0.3$ & 0.978 & NA & NA \\
\hline Clamp insulin (pmol/1) & $441 \pm 81$ & $412 \pm 49$ & $488 \pm 61$ & $453 \pm 69$ & $504 \pm 78$ & $460 \pm 81$ & 0.140 & NA & NA \\
\hline Adiponectin (ng/ml) & $16(12-22)$ & $10(8-12)$ & $10(8-18)$ & $11(7-14)$ & $20(12-23)$ & $12(8-17)$ & 0.689 & NA & $\mathrm{NA}$ \\
\hline Resistin (ng/ml) & $4.5(3.4-5.9)$ & $3.8(3.3-4.4)$ & $4.8(3.9-6.4)$ & $5.1(4.0-6.5)$ & $4.8(3.7-5.6)$ & $6.0(4.4-7.6)$ & 0.015 & 0.07 & 0.01 \\
\hline Leptin (ng/ml) & $1.0(0.6-1.9)$ & $1.3(0.9-1.8)$ & $1.7(0.6-3.6)$ & $1.8(0.6-3.5)$ & $1.6(0.7-3.0)$ & $2.5(0.8-6.5)$ & 0.087 & NA & NA \\
\hline
\end{tabular}

Data are means $\pm \mathrm{SD}$ or median (interquartile range)

Between-group changes from baseline were tested by Kruskal-Wallis with trend analysis $(p 1)$. In case of a significant finding, post hoc testing was done ( $p 2$, prednisolone $7.5 \mathrm{mg}$ vs placebo; $p 3$, prednisolone $30 \mathrm{mg}$ vs placebo)

$\mathrm{AUC}_{\mathrm{GIP}}$, area under the postprandial GIP curve; $\mathrm{AUC}_{\mathrm{GLG}}$, area under the postprandial glucagon curve; AUC $\mathrm{GLP}_{\text {, }}$ area under the postprandial GLP curve; DBP, diastolic blood pressure; FPI, fasting plasma insulin; SBP, systolic blood pressure 
Table 3 Capillary density before and during treatment

\begin{tabular}{|c|c|c|c|c|c|c|c|c|c|}
\hline \multirow[t]{2}{*}{ Variable } & \multicolumn{2}{|l|}{ Placebo } & \multicolumn{2}{|c|}{ Prednisolone $7.5 \mathrm{mg}$} & \multicolumn{2}{|c|}{ Prednisolone $30 \mathrm{mg}$} & \multirow[t]{2}{*}{ pl } & \multirow[t]{2}{*}{$p 2$} & \multirow[t]{2}{*}{ p3 } \\
\hline & Baseline & On treatment & Baseline & On treatment & Baseline & On treatment & & & \\
\hline Baseline density, fasting $\left(\mathrm{n} / \mathrm{mm}^{2}\right)$ & $37.6 \pm 3.9$ & $40.9 \pm 5.1$ & $39.4 \pm 2.9$ & $40.2 \pm 4.3$ & $40.1 \pm 6.3$ & $41.8 \pm 6.6$ & 0.688 & NA & NA \\
\hline Peak density, fasting $\left(\mathrm{n} / \mathrm{mm}^{2}\right)$ & $47.6 \pm 5.4$ & $52.1 \pm 6.0$ & $51.3 \pm 5.6$ & $54.3 \pm 7.1$ & $52.0 \pm 8.9$ & $54.8 \pm 9.8$ & 0.189 & NA & NA \\
\hline Capillary recruitment, fasting (\%) & $26.6 \pm 2.8$ & $26.4 \pm 8.4$ & $30.0 \pm 5.8$ & $30.8 \pm 7.7$ & $29.7 \pm 6.6$ & $28.5 \pm 8.5$ & 1.0 & NA & NA \\
\hline Venous occlusion $\left(\mathrm{n} / \mathrm{mm}^{2}\right)$ & $51.1 \pm 6.1$ & $54.5 \pm 6.4$ & $59.6 \pm 7.8$ & $60.0 \pm 7.6$ & $57.0 \pm 7.9$ & $60.3 \pm 10.6$ & 0.742 & NA & NA \\
\hline Baseline density, hyperinsulinaemia $\left(\mathrm{n} / \mathrm{mm}^{2}\right)$ & $37.1 \pm 2.1$ & $41.4 \pm 6.5$ & $39.3 \pm 4.0$ & $41.0 \pm 5.6$ & $39.1 \pm 6.5$ & $41.7 \pm 7.4$ & 0.701 & NA & NA \\
\hline Peak density, hyperinsulinaemia $\left(\mathrm{n} / \mathrm{mm}^{2}\right)$ & $51.3 \pm 2.2$ & $60.8 \pm 8.1$ & $57.1 \pm 8.0$ & $57.9 \pm 8.4$ & $55.1 \pm 9.7$ & $54.4 \pm 10.3$ & 0.011 & 0.03 & 0.038 \\
\hline Capillary recruitment, hyperinsulinaemia (\%) & $38.7 \pm 4.2$ & $44.8 \pm 9.8$ & $44.9 \pm 8.2$ & $41.5 \pm 12.9$ & $41.2 \pm 5.2$ & $30.6 \pm 8.2$ & 0.001 & 0.18 & 0.002 \\
\hline
\end{tabular}

Data are means $\pm \mathrm{SD}$

Between-group changes from baseline were tested by Kruskal-Wallis with trend analysis $(p 1)$. In case of a significant finding, post hoc testing was done ( $p 2$, prednisolone $7.5 \mathrm{mg}$ vs placebo; $p 3$, prednisolone $30 \mathrm{mg}$ vs placebo)

$38 \%$ of the prednisolone-induced changes in insulin-mediated glucose disposal ( $\beta$ changed from -0.695 to -0.434 ). Finally, prednisolone-induced changes in insulin-stimulated capillary recruitment were negatively correlated with the observed rise in systolic blood pressure (Fig. 3e).
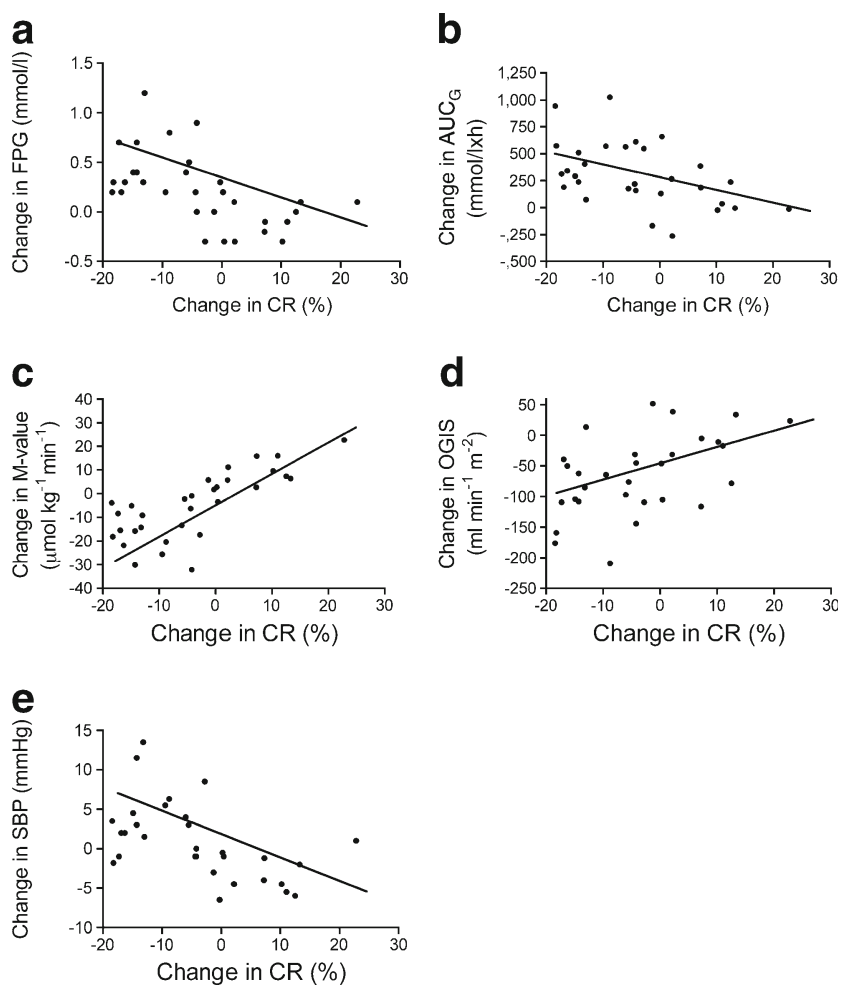

Fig. 3 Association between prednisolone-induced changes in insulinstimulated microvascular function (CR) and prednisolone-induced changes in metabolic and vascular variables, including FPG $(r=-0.64$; $p<0.001)(\mathbf{a}), \mathrm{AUC}_{\mathrm{G}}(r=-0.52 ; p=0.003)(\mathbf{b})$, insulin-stimulated glucose disposal $(r=+0.76 ; p<0.001)(\mathbf{c})$, postprandial insulin sensitivity $(r=+0.51 ; p=0.004)(\mathbf{d})$ and systolic blood pressure (SBP) $(r=-0.62$; $p<0.001)(\mathbf{e})$
Prednisolone-induced increment in resistin levels are related to impaired microvascular function Prednisolone dose-dependently increased resistin levels, whereas the concentration of the adipokines adiponectin and leptin were not significantly altered by the study medication (Table 2, Fig. 4a). The change in resistin level was significantly related to the prednisolone-induced impairment of insulin-stimulated capillary recruitment (Fig. 4b).

Safety and tolerability One participant in the prednisolone $30 \mathrm{mg}$ group complained of sleeplessness, which was of a mild and transient nature. Otherwise, no side effects were reported in any treatment arm.

\section{Discussion}

This is the first study describing a dose-dependent impairment of insulin-stimulated microvascular function following GC treatment in healthy individuals as a possible mechanism contributing to the concomitantly occurring decrease in a

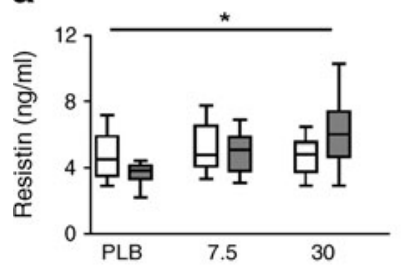

b

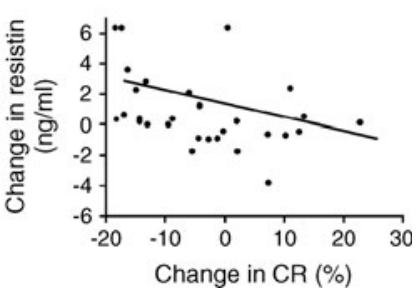

Fig. 4 Prednisolone treatment dose-dependently increased plasma resistin concentrations (a) and this was associated $(r=-0.397 ; p=0.03)$ with the decrease in insulin-stimulated capillary recruitment (CR) (b). Median and interquartile range are shown. White bar, pre-treatment; grey bar, following 2 weeks of prednisolone treatment; PLB, placebo; 7.5, prednisolone $7.5 \mathrm{mg}$ once daily; 30 , prednisolone $30 \mathrm{mg}$ once daily. $* p<0.05$ across groups, change from baseline 
insulin sensitivity and glucose tolerance and elevation of blood pressure. Furthermore, we observed a correlation between changes in plasma concentrations of the adipokine resistin and the impairment in insulin-stimulated capillary recruitment.

It has been reported previously that GCs particularly impair metabolism under stimulated conditions (i.e. during hyperinsulinaemic-euglycaemic clamps or in the postprandial state). Accordingly, we have recently shown that prednisolone dose-dependently impaired metabolic fluxes during insulin infusion, but not in the fasted state when prednisolone induced fasting hyperinsulinaemia [6]. Moreover, fasting glucose levels are often only mildly elevated by GC treatment, whereas during the day significant hyperglycaemia develops, especially in the postprandial period [21,24]. This seems also to be the case for the vascular effects of prednisolone, because in the present study we did not observe prednisolone-induced changes in capillary recruitment in the fasted state, when prednisolone augmented fasting insulin levels. Collectively, the data demonstrated that prednisolone induced vascular insulin resistance.

Subsequently, we analysed whether vascular insulin resistance was related to the prednisolone-induced impairment of glucose metabolism and increase in blood pressure. Indeed, prednisolone-induced impairment in capillary recruitment was significantly associated with insulin-stimulated glucose disposal during the hyperinsulinaemic-euglycaemic clamp, as well as with postprandial glucose levels and postprandial insulin sensitivity. This suggests that capillary recruitment may also be an important factor contributing to postprandial glucose metabolism. In line with this finding, Keske and colleagues recently demonstrated, using contrast-enhanced ultrasound, that in obese insulin-resistant individuals, capillary recruitment was impaired in the postprandial state [25], which was more recently also shown for type 2 diabetic patients [26]. Additionally, the approximately $6 \mathrm{mmHg}$ increase in systolic blood pressure induced by high-dose prednisolone treatment was related to the decrease in insulinstimulated capillary recruitment. Previously, various mechanisms have been proposed to underlie the GC-induced increase in blood pressure, including salt and water retention, altered sympathovagal balance, increased responsiveness to pressor stimuli (such as catecholamines and angiotensin II) and reduced NO availability [27]. We suggest that GCinduced insulin resistance at the level of the microcirculation may be an alternative mechanism to explain the increase in blood pressure observed during GC treatment.

In recent years, the role of altered adipose tissue function, including perivascular adipose tissue, has received attention in the pathogenesis of microvascular dysfunction $[15,17,28]$. By secreting various adipokines, including hormones, cytokines and chemokines, adipose tissue and macrophages residing in adipose tissue were shown to influence microvascular function in a mouse model of type 2 diabetes [28]. Interestingly, GCs display extensive effects on adipose tissue: GCs increase total fat mass, induce adipose tissue insulin resistance resulting in increased lipolysis [6] and alter the secretion of a number of adipokines in vitro towards a more diabetogenic profile $[3,7]$. These alterations are characterised by increased expression of resistin and leptin and are associated with insulin resistance and reduced concentrations of adiponectin $[3,7]$. On the other hand, GCs decrease pro-inflammatory cytokines, including TNF- $\alpha$, and C-reactive protein through their antiinflammatory effects. Therefore we studied the effects of GCs on the adipokines adiponectin, leptin and resistin, since they may be altered by GC treatment and are to known to alter microvascular function [17]. Prednisolone treatment did not significantly alter adiponectin and leptin concentrations but it did dose-dependently increase plasma resistin concentrations. Interestingly, the change in resistin level was related to the reduction in capillary recruitment. In human coronary artery endothelial cells, resistin was shown to impair endothelial function by reducing NO synthesis [29]. In C57BL/6 mice, resistin impaired insulin-evoked vasodilatation by reducing eNOS activity [30]. The effects of resistin were specific for the action of insulin, since acetylcholine-induced vasodilatation was not affected [30]. Our data may point towards a role for resistin in the development of GC-induced microvascular dysfunction. This hypothesis will need to be further addressed in mechanistic studies.

Since muscle tissue is the main peripheral site of insulinmediated glucose uptake and vascular resistance, it would have been more straightforward to measure insulin-mediated microvascular recruitment in muscle instead of skin. However, the human skin is the only site available in humans to directly and non-invasively assess capillary density and recruitment of capillaries. Moreover, the effects of obesity and NEFA on insulin-mediated microvascular recruitment in muscle [31, 32] can be reproduced in human skin [18, 33] suggesting that the vascular responses observed in skin reflect those in muscle. In a recent study, we could demonstrate concurrent insulin-mediated microvascular recruitment in skin and skeletal muscle [34]. Both were mutually related and strongly associated with whole-body glucose uptake suggesting that the cutaneous microcirculation is a representative vascular bed to examine insulin's actions on the microcirculation.

Limitations of our study include a relatively small study size and our study population consisting of healthy individuals. Although this population allows the metabolic and microvascular effects of prednisolone to be studied without interfering factors such as pre-existent diseases or use of medications, in the clinical setting prednisolone is used to treat patients with chronic inflammatory diseases. As systemic inflammation also impairs microvascular function [35], the effects of GCs on microvascular function and insulin 
sensitivity, given their combined anti-inflammatory and metabolic effects, could be more complex in this population.

In conclusion, prednisolone treatment in healthy individuals dose-dependently impaired insulin-stimulated capillary recruitment. In addition, prednisolone-induced changes in insulin-stimulated capillary recruitment were strongly related to unfavourable metabolic effects induced by prednisolone treatment, including increased postprandial glycaemic excursions and decreased insulin sensitivity as well as increased systolic blood pressure. Importantly, a substantial part of the decrease in insulin-stimulated glucose disposal could be explained by prednisolone-induced microvascular dysfunction. Finally, following prednisolone treatment we observed increased plasma concentrations of the adipokine resistin and this increase was related to impaired microvascular function. We propose that vascular insulin resistance, in addition to pancreatic alpha and beta cell dysfunction $[3,21]$ and insulin resistance at the level of adipose tissue, liver and skeletal muscle [6], may contribute to the metabolic side effects associated with GC treatment.

Funding This paper was written within the framework of the Dutch Top Institute Pharma project T1-106.

Duality of interest The authors declare that there is no duality of interest associated with this manuscript.

Contribution statement All authors made substantial contributions to conception and design, acquisition of data, or analysis and interpretation of data. They were all involved in writing the manuscript and approved the final version to be published.

\section{References}

1. Gulliford MC, Charlton J, Latinovic R (2006) Risk of diabetes associated with prescribed glucocorticoids in a large population. Diabetes Care 29:2728-2729

2. Schacke H, Docke WD, Asadullah K (2002) Mechanisms involved in the side effects of glucocorticoids. Pharmacol Ther 96:23-43

3. van Raalte DH, Ouwens DM, Diamant M (2009) Novel insights into glucocorticoid-mediated diabetogenic effects: towards expansion of therapeutic options? Eur J Clin Invest 39:81-93

4. Walker BR (2007) Glucocorticoids and cardiovascular disease. Eur J Endocrinol 157:545-559

5. Williamson PM, Kelly JJ, Whitworth JA (1996) Dose-response relationships and mineralocorticoid activity in cortisol-induced hypertension in humans. J Hypertens 14(Suppl):S37-S41

6. van Raalte DH, Brands M, van der Zijl NJ et al (2011) Low-dose glucocorticoid treatment affects multiple aspects of intermediary metabolism in healthy humans: a randomised controlled trial. Diabetologia 54:2103-2112

7. Fasshauer M, Paschke R (2003) Regulation of adipocytokines and insulin resistance. Diabetologia 46:1594-1603

8. Saltiel AR, Kahn CR (2001) Insulin signalling and the regulation of glucose and lipid metabolism. Nature 414:799-806
9. Barrett EJ, Eggleston EM, Inyard AC et al (2009) The vascular actions of insulin control its delivery to muscle and regulate the rate-limiting step in skeletal muscle insulin action. Diabetologia 52:752-764

10. Kubota T, Kubota N, Kumagai H et al (2011) Impaired insulin signaling in endothelial cells reduces insulin-induced glucose uptake by skeletal muscle. Cell Metab 13:294-307

11. Serne EH, de Jongh RT, Eringa EC, Ijzerman RG (2007) Microvascular dysfunction: a potential pathophysiological role in the metabolic syndrome. Hypertension 50:204-211

12. Kim JA, Montagnani M, Koh KK, Quon MJ (2006) Reciprocal relationships between insulin resistance and endothelial dysfunction: molecular and pathophysiological mechanisms. Circulation 113:1888-1904

13. Li R, Zhang H, Wang W et al (2010) Vascular insulin resistance in prehypertensive rats: role of PI3-kinase/Akt/eNOS signaling. Eur J Pharmacol 628:140-147

14. Muniyappa R, Montagnani M, Koh KK, Quon MJ (2007) Cardiovascular actions of insulin. Endocr Rev 28:463-491

15. Bakker W, Eringa EC, Sipkema P, van Hinsbergh VW (2009) Endothelial dysfunction and diabetes: roles of hyperglycemia, impaired insulin signaling and obesity. Cell Tissue Res 335:165-189

16. Eringa EC, Bakker W, Smulders YM, Serne EH, Yudkin JS, Stehouwer CD (2007) Regulation of vascular function and insulin sensitivity by adipose tissue: focus on perivascular adipose tissue. Microcirculation 14:389-402

17. Meijer RI, Serne EH, Smulders YM, van Hinsbergh VW, Yudkin JS, Eringa EC (2011) Perivascular adipose tissue and its role in type 2 diabetes and cardiovascular disease. Curr Diab Rep 11:211-217

18. de Jongh RT, Serne EH, Ijzerman RG, de Vries G, Stehouwer CD (2004) Impaired microvascular function in obesity: implications for obesity-associated microangiopathy, hypertension, and insulin resistance. Circulation 109:2529-2535

19. DeFronzo RA, Tobin JD, Andres R (1979) Glucose clamp technique: a method for quantifying insulin secretion and resistance. Am J Physiol 237:E214-E223

20. Mari A, Pacini G, Murphy E, Ludvik B, Nolan JJ (2001) A modelbased method for assessing insulin sensitivity from the oral glucose tolerance test. Diabetes Care 24:539-548

21. van Raalte DH, Nofrate V, Bunck MC et al (2010) Acute and 2-week exposure to prednisolone impair different aspects of beta-cell function in healthy men. Eur J Endocrinol 162:729-735

22. Orskov C, Rabenhoj L, Wettergren A, Kofod H, Holst JJ (1994) Tissue and plasma concentrations of amidated and glycine-extended glucagon-like peptide I in humans. Diabetes 43:535-539

23. Deacon CF, Nauck MA, Meier J, Hucking K, Holst JJ (2000) Degradation of endogenous and exogenous gastric inhibitory polypeptide in healthy and in type 2 diabetic subjects as revealed using a new assay for the intact peptide. J Clin Endocrinol Metab 85:3575-3581

24. Clore JN, Thurby-Hay L (2009) Glucocorticoid-induced hyperglycemia. Endocr Pract 15:469-474

25. Keske MA, Clerk LH, Price WJ, Jahn LA, Barrett EJ (2009) Obesity blunts microvascular recruitment in human forearm muscle after a mixed meal. Diabetes Care 32:1672-1677

26. van Genugten RE, Serne EH, Heymans MW, van Raalte DH, Diamant M (2013) Postprandial microvascular function deteriorates in parallel with gradual worsening of insulin sensitivity and glucose tolerance in men with the metabolic syndrome or type 2 diabetes. Diabetologia 56:583-587

27. Whitworth JA, Schyvens CG, Zhang Y, Mangos GJ, Kelly JJ (2001) Glucocorticoid-induced hypertension: from mouse to man. Clin Exp Pharmacol Physiol 28:993-996

28. Meijer RI, Bakker W, Alta CL et al (2013) Perivascular adipose tissue control of insulin-induced vasoreactivity in muscle is impaired in $\mathrm{db} /$ $\mathrm{db}$ mice. Diabetes 62:590-598 
29. Chen C, Jiang J, Lu JM et al (2010) Resistin decreases expression of endothelial nitric oxide synthase through oxidative stress in human coronary artery endothelial cells. Am J Physiol Heart Circ Physiol 299:H193-H201

30. Gentile MT, Vecchione C, Marino G et al (2008) Resistin impairs insulin-evoked vasodilation. Diabetes 57:577-583

31. Liu Z, Liu J, Jahn LA, Fowler DE, Barrett EJ (2009) Infusing lipid raises plasma free fatty acids and induces insulin resistance in muscle microvasculature. J Clin Endocrinol Metab 94:3543-3549

32. Clerk LH, Vincent MA, Jahn LA, Liu Z, Lindner JR, Barrett EJ (2006) Obesity blunts insulin-mediated microvascular recruitment in human forearm muscle. Diabetes 55:1436-1442
33. de Jongh RT, Serne EH, Ijzerman RG, de Vries G, Stehouwer CD (2004) Free fatty acid levels modulate microvascular function: relevance for obesity-associated insulin resistance, hypertension, and microangiopathy. Diabetes 53:2873-2882

34. Meijer RI, de Boer MP, Groen MR et al (2012) Insulin-induced microvascular recruitment in skin and muscle are related and both are associated with whole-body glucose uptake. Microcirculation 19:494-500

35. Eringa EC, Stehouwer CD, Walburg K et al (2006) Physiological concentrations of insulin induce endothelin-dependent vasoconstriction of skeletal muscle resistance arteries in the presence of tumor necrosis factor-alpha dependence on c-Jun $\mathrm{N}$-terminal kinase. Arterioscler Thromb Vasc Biol 26:274-280 